\title{
Clinical assessment in primary care: professional cooperation
}

\author{
B Y VIVIEN REID \\ Department of Preventive Medicine/Cardiology, Education and Research Centre, \\ St Vincent's Hospital, Elm Park, Dublin 4, Republic of Ireland
}

The late Dr Denis Burkitt, well known to nutritionists for his work on high-fibre diets in the prevention of bowel disease, compared illness with an overflowing bath and suggested that doctors and nurses might be better employed turning off the taps rather than mopping the floor. It is not enough just to turn off the taps, we need to make sure they don't get turned on again.

There is increasing interest in, and commitment to, prevention of disease and health promotion in primary care. In the UK health promotion was made a contractual requirement for general practitioners (GP) in 1990. In the Republic of Ireland, the recently-published health strategy document, Shaping a Healthier Future (Department of Health, 1994), has identified preventive medicine as an area for expansion in general practice. In the followon document to the health strategy document, A Health Promotion Strategy (Department of Health, 1995a), GP are identified as having 'a key role in health promotion in terms of expertise, numbers and level of contact with the public'. Up to the present time, health promotion activities have not been directly funded in general practice in the Republic of Ireland.

In order to personalize health promotion in primary care, i.e. to employ preventive medicine, factors that increase risk of ill health must be identified. There is ongoing debate as to the effectiveness of clinical assessment irrespective of risk status. There are advocates of high-risk-strategy intervention and others who hold that even those identified not to be at high risk may benefit from clinical assessment and basic health information.

A number of published studies have been carried out in the UK, Canada and Sweden which have evaluated health screening and intervention in primary care. Preliminary programmes are under way in the Republic of Ireland against a background of health screening and intervention which are not funded in general practice.

\section{CLINICAL ASSESSMENT IN PRIMARY CARE: LOCAL INITIATIVES The Coronary Risk Intervention Strategy Programme (CRISP)}

CRISP was initiated by the Department of Preventive Medicine/Cardiology, St Vincent's Hospital, Dublin in 1990 to help develop health assessment and prevention by health promotion in the general-practice setting. It was recognized that risk identification should not be undertaken unless an adequate programme for risk-factor modification was in place. In addition, the need to audit such programmes was recognized in order that the most effective and efficient service would be provided.

At that time, the Kilkenny Health Project, in conjunction with the Kilkenny Faculty of the Irish College of General Practitioners, had already established that a health assessment programme was possible in general practice (Kilkenny Health Project, 1992). It remained to be seen whether subsequent counselling and management could achieve a worthwhile 
reduction in cardiovascular risk factors. The Irish Heart Foundation (1994) was also initiating its community-based Happy Heart Programme which aims to involve local communities in heart disease prevention throughout the country. CRISP was seen as a possible adjunct to this, providing personal risk assessment and intervention in general practice to complement the community-based information in the Happy Heart Programme.

The pilot programme of CRISP was undertaken in two large group practices in the catchment area of the hospital during 1990 and 1991. The main outcome of the pilot programme related to the practical aspects of carrying it out. The two practices differed: in one health assessment and intervention was provided by the doctor, which proved to be very timeconsuming; in the other practice health assessment and intervention was provided by the practice nurse in association with the doctor. From the pilot programme, the practice nurse was identified to be an essential resource for the implementation of CRISP. The costs involved in health assessment were borne by the practice, as this was not a centrally-funded activity in general practice. Additional costs were accrued for cholesterol testing. Nutrition and dietetic advice is not funded in general practice and patients were required to pay for this service where it was available. Of the patients assessed, less that $50 \%$ were patients who could be charged for the service, the rest were entitled to be treated free of charge due to their low income.

Following the pilot programme a decision was made to establish CRISP more widely with these supports:

for practices without a nurse, a sessional nurse would be provided to conduct the assessment of patients on-site in the practice and to be involved in some aspects of counselling; the sessional nurse would provide a Lipotrend Analyser (Boehringer, Mannheim, Germany) for cholesterol estimation and the running costs would be subsidized. Practices with their own nurse and equipment would have costs subsidized;

back-up dietetic counselling in the practices would be provided;

referral of patients where needed to the Cardiovascular Risk-factor Intervention Clinic at the hospital would be facilitated;

patients for CRISP assessment would be identified by practice staff and be given an appointment with the sessional or practice nurse.

The aim was to involve approximately twelve practices in the catchment area of the hospital, including both group practices and single-handed GP. In addition, similar practices in County Wexford were to be included in CRISP, in conjunction with the local hospital and the Happy Heart Programme in the area.

\section{OUTCOME OF CORONARY RISK INTERVENTION STRATEGY PROGRAMME AUDIT DECEMBER 1994}

Nineteen practices participated in the programme from 1992 to 1994. Nine practices were in the catchment area of the hospital, nine were in County Wexford and one was in the south midlands and had requested to participate in the programme.

A total of 1813 patients had a health assessment in their practice, carried out by the practice nurse, or the CRISP nurse provided on a sessional basis by the Department of Preventive Medicine/Cardiology, St Vincent's Hospital. Seventeen practices were provided with a CRISP nurse. All the nurses received training in the implementation of CRISP, including completing the assessment form, making the required measurements, and coun- 
Table 1. Initial assessment of the Coronary Risk Intervention Strategy Programme* (\% of total sample)

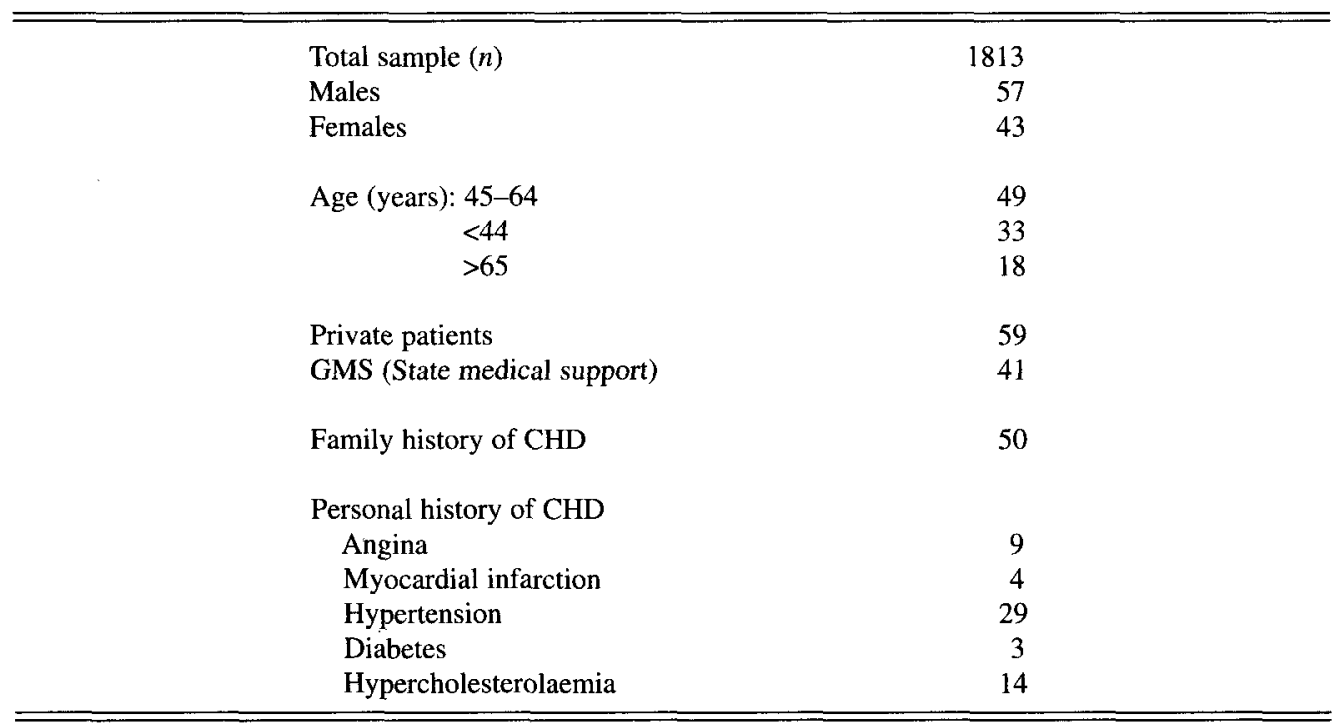

* For details of programme, see p. 679.

selling patients to make appropriate lifestyle changes. Department of Preventive Medicine/Cardiology staff involved in the training included medical and nursing staff and a dietitian.

Details of the initial assessment data are presented in Table 1. There were more males than females and half the patients were in the age-group 45-64 years. Private patients comprised more than half the patients. Family history of CHD was recorded in more than half the patients. There was a personal history of hypertension in $29 \%$ of patients and of hypercholesterolaemia in $14 \%$ of cases. The initial risk-factor profile is presented in Table 2 . Of those assessed $26 \%$ were cigarette smokers with an additional $5 \%$ cigar smokers, $23 \%$ were identified as having a cholesterol level greater than $6.4 \mathrm{mmol} / \mathrm{l}$ on Lipotrend analysis, $65 \%$ were in the overweight categories of which $22 \%$ were obese, $48 \%$ had unacceptably-low exercise levels, $11 \%$ drank more than the recommended intake of alcohol and $11 \%$ of patients had previously been prescribed a therapeutic diet.

Following initial assessment in the practice, counselling was provided by the trained nurse to facilitate long-term change in health risk. A number of other initiatives were put in place by the Department of Preventive Medicine/Cardiology to facilitate these changes.

\section{Training days}

These were provided on a regular basis to give ongoing support to the practice nurses. They were implemented by the CRISP nurse facilitator and the CRISP dietitian.

\section{'Stop smoking' training days}

These were established to train health professionals in the techniques required to help smokers to stop. These training days also provided information on group support and on setting up 6-week stop smoking courses. 
Table 2. Initial risk factor profile for the Coronary Risk Intervention Strategy Programme* (\% total sample $\dagger$ )

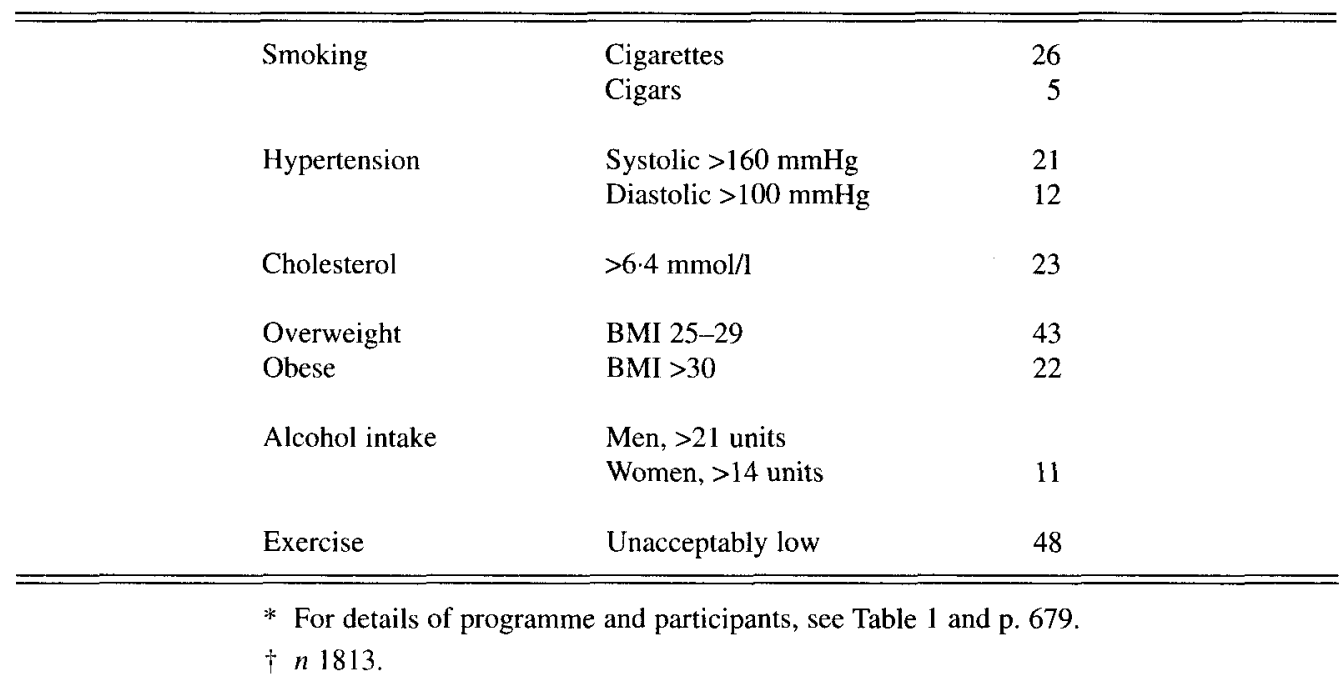

\section{Community School adult education courses}

On the request of a GP participating in CRISP, a 10-week 'healthy cookery' course was established in a local community school as part of the adult education programme. This involved a cookery expert interested in healthy eating, the GP and the dietitian from the Department of Preventive Medicine/Cardiology. It was a cookery demonstration course which provided practical skills to put healthy eating into practice. The course has since been extended to a second community school and is an appropriate model for implementation throughout the country.

A 6-week 'stop smoking' course was also established in the community school as an adult education course and has since been extended to other community schools. The 'stop smoking' training days also provided the necessary training for interested health professionals to establish these courses in local community schools.

\section{'Stop smoking' courses}

These were provided at St Vincent's Hospital on a regular basis. A drop-in centre was available each month to smokers who needed help to stop. Smokers who had been identified in CRISP and needed help to stop smoking could be referred to these sessions.

An information card publicizing these resources was produced in conjunction with the Health Promotion Unit at the Department of Health.

\section{FOLLOW-UP DATA}

At initial assessment of the 1813 patients, $1200(66 \%)$ were identified as requiring followup. Of that 1200 , only 422 patients returned for follow-up at 6 months, i.e. $35 \%$ of those requiring follow-up and $23 \%$ of those who had an initial assessment. Details of the changes in risk factors are shown in Table 3 . Disappointingly, only $2 \%$ of patients stopped smoking, which may be a reflection of the small numbers who recorded having attended a 'stop 
Table 3. Change in risk factor profile for the Coronary Risk Intervention Strategy Programme* for 422 patients at 6-month follow-up

\begin{tabular}{|c|c|}
\hline Smoking & $\Downarrow 2 \%$ \\
\hline $\begin{aligned} \text { Hypertension: } & \text { Systolic } \\
& \text { Diastolic }\end{aligned}$ & $\begin{array}{l}\Downarrow 19 \mathrm{mmHg} \\
\Downarrow 8 \mathrm{mmHg}\end{array}$ \\
\hline Cholesterol & $\Downarrow 0.7 \mathrm{mmol} / /$ \\
\hline $\begin{array}{l}\text { Overweight } \\
\text { Obese }\end{array}$ & $\begin{array}{l}\Downarrow 0.8 \mathrm{~kg} \\
\Downarrow 1.7 \mathrm{~kg}\end{array}$ \\
\hline Alcohol intake high & $47 \%$ reduced \\
\hline Exercise unacceptably low & $44 \%$ increased \\
\hline
\end{tabular}

smoking' programme, which were generally not available at practice level. Changes in cholesterol levels and weight were small. Only $3.5 \%$ of patients attended a group weightmanagement programme and $14 \%$ attended for individual dietary counselling. This reflects the lack of dietetic services for patients at practice level. Alcohol intakes were reported to have been reduced and exercise levels increased by those who had unacceptable levels recorded at initial assessment.

\section{DISCUSSION}

A limitation of CRISP arose from the provision of sessional nurses to practices, where they did not become an integral part of the practice. Although the nurses were well trained and enthusiastic about their work, they were often restrained by practice commitment and organizational limitations outside their control. However, a number of the practices involved in CRISP have recently employed practice nurses. The two pilot practices continue to use CRISP and have now been computerized as part of their inclusion as pilot practices for the evaluation of developments in general practice by the Eastern Health Board.

As in all aspects of health care, there are differences in how services operate for a variety of reasons. One practice which participated in CRISP showed particular enthusiasm for the programme by asking to participate in it and by following through in its implementation (with minimal support from the Department of Preventive Medicine/Cardiology, as it was outside the geographical areas we had designated initially). The practice is a group practice of three GP and a practice nurse. A dietitian provides a service to the practice once monthly.

Initial CRISP assessment of patients in this practice revealed a large proportion of patients with a family and personal history of CHD. Follow-up was recommended for $82 \%$ of those assessed and 43\% of these patients returned for 6-month follow-up. The low return rate for follow-up in the CRISP audit (35\%) highlights the difficulty of ongoing health promotion in this setting. Poor attendance rates may reduce the impact of the intervention; however, health checks provide only one aspect of health promotion in primary care (Imperial Cancer Research Fund OXCHECK Study Group, 1995). In this practice, support 
was provided for smokers by individual counselling and group sessions. This was reflected in more smokers giving up (7\%) than in CRISP $(2 \%)$.

Participation of CRISP patients in the Community School adult education courses was disappointing. GP need to encourage patients to avail themselves of these supports. All adult education courses are funded by the participants themselves, therefore, some subsidy from Health Boards may be necessary to encourage those who could benefit from this type of support to attend.

As part of CRISP, the provision of back-up dietetic counselling in participating practices was planned. Due to limited funding it was not possible to provide an open-ended service. Efforts were made with local dietitians to assist in the development of services, but this was inhibited by the lack of central funding. With support from the Health Promotion Unit at the Department of Health it was possible to employ a CRISP dietitian for $1 \mathrm{~d} /$ week at the Department of Preventive Medicine/Cardiology to participate in the practice nurse training programmes. The dietitian was also available to the nurses for consultation. In one large group practice the CRISP dietitian initiated a 'Be a healthy weight' course. Each course ran for four consecutive weeks with a $1 \mathrm{~h}$ session each week. Counselling and support were offered to those who were concerned about their weight and its effect on their health. The course provided dietary assessment by the dietitian using food diaries and a contract for change was drawn up with each participant. Six people participated in each course and all benefited from it. The participation of a GP in one of the four sessions in the course added greatly to its impact. Careful selection of patients by the practice nurse and GP, with the dietitian's guidance, was essential to facilitate maximum benefit from the course. We hope that this approach to dietary intervention in general practice may be a useful model for the future. The CRISP dietitian participated in the 'stop smoking' courses, advising clients on weight management when stopping smoking. In conjunction with this a handout was prepared and supported by the Health Promotion Unit entitled Stop Smoking the Slim Way. She participated in the 'healthy cookery' course in community schools, providing training in nutrition for the demonstrator and practical advice to those who participated in the course.

A series of twelve articles on practical aspects of nutrition and dietetics were written by the CRISP dietitian and published in Forum, the Journal of the Irish College of General Practitioners. These were well received by GP and future articles by specialist dietitians will be coordinated by that dietitian. Plans are in place to publish these articles in book form as a practical reference for health professionals in general practice.

As with the practice nurse, the integration of the dietitian as a member of the practice team is essential for maximum benefit to the practice and ultimately the patients. The dietitian can provide support to the GP and the practice nurse in keeping up to date with nutrition developments and in providing practical approaches to implementing dietary change. Integration of the dietitian into the practice will also help widen the range of referrals for dietary advice; overweight patients are the main referrals in most practices (O. Carolan, personal communication). Clinical assessment of patients will identify additional need for referral. With a dietitian attached to the practice, nutritional assessment (in questionnaire form) may assist in identifying those needing referral. Patients with diabetes and those at high risk for CHD can also be managed more adequately in the practice with the input of the dietitian. The management of feeding problems in children, constipation, Fe-deficiency anaemia, hyperlipidaemia and the implementation of general healthylifestyle advice also requires the input of the dietitian (Gleeson \& Flynn, 1990). 
With recent developments in the employment of dietitians through GP units in some Health Board Areas to provide services to general practice, there is an urgent need for evaluation of methods of practice outside the clinical-care setting. We must be open to alternative methods of communicating and implementing health promotion advice.

\section{A HEALTH PROMOTION INTERVENTION PROGRAMME IN GENERAL PRACTICE FOR THE PREVENTION OF CARDIOVASCULAR DISEASE IN CHILDREN}

This health promotion intervention programme is being carried out by the Department of Health Promotion at University College, Galway, as part of the Galway Health Project. There is collaboration with GP and schools to implement primary prevention of cardiovascular disease among schoolchildren aged 8-15 years and their families. Traditionally cardiovascular disease prevention programmes have focused on adult risk factors and risk behaviour. However, the British Family Heart Study has shown a 16\% reduction in coronary risk score using a family-oriented prevention programme (Family Heart Study Group, 1994).

This study sets out to evaluate the time, personnel and resources needed for health promotion in general practice. It will evaluate also the opportunities for an approach to health promotion to children aged 8-15 years, integrated between schools and general practice. A lifestyle questionnaire will be completed by children and their parents before interview. Specific health outcome indicators will also be measured: increase in knowledge about healthy diet and exercise, a reduction in the number of smokers and an increase in those engaging in regular physical activity. The pilot study of this programme, carried out at the end of 1994, indicated the need to plan health promotion initiatives rather than depending on an opportunistic approach. The input of a practice nurse also made health promotion in the practice more feasible.

A baseline survey of health promotion practices in a sample of schools in the Galway area was undertaken in Spring 1995. A Health Promoting School model will be followed to implement health promotion, involving curriculum review, the school environment and the links between the school and the community. The recruitment of general practices to participate in the study is currently under way. The baseline lifestyle questionnaire is being prepared and suitable health promotion materials are being developed in association with the community nutritionist. We await with interest the outcome of this study which will have implications for the future implementation of health promotion activities in general practice.

\section{CLINICAL ASSESSMENT IN PRIMARY CARE: PUBLISHED STUDIES}

\section{The British Family Heart Study}

This was a randomized controlled trial in twenty-six general practices in Britain (Family Heart Study Group, 1994). The objective was to measure the change in cardiovascular risk factors achievable in families over 1 year by cardiovascular screening and lifestyle intervention in general practice. This was a practice-nurse-led programme using a familycentred approach with follow-up according to degrees of risk. After 1 year (a) coronary risk score and (b) cigarette smoking, weight, blood pressure and random blood cholesterol and glucose concentrations were compared for the intervention and control practices. 
In the intervention practices at 1 year, reduction in coronary risk score was $16 \%$. There was a $4 \%$ reduction in smoking with small changes in weight, cholesterol and blood pressure. Reduction in risk factors was greatest for highest initial levels of these variables. Longterm maintenance of these changes in risk factors would mean a $12 \%$ reduction in risk of coronary events. It was noted that $88 \%$ of men and $85 \%$ of women returned for the 1 -year follow-up. With lower return rates in CRISP, a $2 \%$ reduction in smoking was recorded at the 6-month follow-up in conjunction with small changes in other risk factors, similar to those in the British Family Heart Study (1994). The authors of the study question the costeffectiveness of this type of intervention and recommend evaluation of other approaches to health screening and intervention.

\section{OXCHECK}

This was a randomized controlled trial in five urban practices in Bedfordshire (Imperial Cancer Research Fund OXCHECK Study Group, 1995). The objective was to assess the effectiveness of health checks performed by nurses in primary care in reducing risk factors for cardiovascular disease and cancer. After 3 years, serum total cholesterol concentration, blood pressure, BMI, and smoking prevalence were measured. Self-reported dietary, exercise and alcohol habits were recorded. This study has shown that health checks by nurses in primary care can lead to sustained changes in dietary behaviour and a reduction of about $3 \%$ in serum cholesterol, with little effect on blood pressure, smoking and alcohol. Annual checks were found to be no more effective for changing risk factors than a single check at 3 years. Unlike CRISP and the British Family Heart Study, OXCHECK showed little reduction in smoking rates. This may result from a greater concentration on dietary intervention, which was reflected in a decrease in serum cholesterol levels. The authors suggest that the benefits of systematic health promotion in primary care are real, but must be weighed against the costs in relation to other priorities.

There has been a certain amount of pessimism about the results of the British Family Heart Study and the OXCHECK Study. Some, however, would hold that these results are acceptable and can yield even better outcomes if such programmes continue to be developed in general practice. Against a background of limited resources, the results support the merit of adopting a preventive approach to CHD risk reduction through primary care that is aimed at all patients and not those judged to be at high risk (Gill, 1995). The inclusion of other health professionals in the primary care team, e.g. dietitians, physiotherapists, psychologists, food and cookery advisors, may be necessary to facilitate the implementation of long-lasting lifestyle changes.

\section{Other studies}

The CELL Study Group in Sweden (Lindholm et al. 1995) have reported that limited additional benefit was gained from participation in group sessions for intensive health care advice, by subjects who had multiple risk factors for cardiovascular disease. The authors suggest that it is difficult to make an important impact on cardiovascular risk in primary care by using only the practice staff (doctors and nurses) and that better methods of communicating the messages need to be devised.

A study carried out in Belfast (Cupples \& McKnight, 1994) which examined the impact of personal health education in a general-practice setting on patients with angina showed 
an improved quality of life for those patients, as well as improved dietary habits and increased frequency of physical exercise. No improvement was seen in blood pressure, cholesterol concentration or rate of stopping smoking at the 2-year follow-up. Looking at the cost-effectiveness of screening and intervention strategies for preventing CHD, Field et al. (1995) report that the most cost-effective strategy is to target patients with raised blood pressure or a history of CHD.

A Canadian study (Grover et al. 1995) concluded that despite guidelines on targeting patients at high risk of coronary disease, accurate assessment of coronary risk remains difficult for many doctors. The publication of the European Society of Cardiology guidelines for the prevention of CHD in clinical practice (Pyorala et al. 1994) has provided an 'easyto-use' risk chart to assist risk assessment. The Clinical Standards Committee of the Irish College of General Practitioners is currently developing practical strategies to implement the guidelines issued in the report.

One must beware of reading too much into study results. Populations vary considerably as to practices in health care. Prevention may be of low priority in some groups for very good reasons. Generalizing from Britain to Sweden to Ireland may not be possible (Toon, 1995). Despite these reservations, efforts must continue to determine which assessment and health promotion strategies work best. It is important to recognize that the relationship between a patient's knowledge, attitudes and behaviour is complex and needs to be taken into account when planning any strategy for preventing disease (Silagy et al. 1993). A further challenge for primary care is to motivate people to change their behaviour. There is also the question of the readiness of the patient to receive and act on lifestyle advice. Marteau et al. (1995) documented significant differences between the ways in which patients view their risk of CHD and their documented risk based on an epidemiologicallyderived index of risk. Effective management is likely to involve the health professional in using both self-assessed and epidemiologically-derived risk scores as the basis for a dialogue with patients on what factors are contributing to their risks of CHD. Factors potentially amenable to modification can then be identified and readiness to consider change can be explored. Consideration must be given to the current method of targeting intervention strategies at individuals. Other strategies may be equally, or even more, effective. These could include group education in schools, workplaces, group general practices and through the media. Government action such as adjusting fiscal and agricultural policy in line with the Recommendation for a Food and Nutrition Policy for Ireland (Department of Health, 1995b), introducing stricter rules on tobacco advertising and food labelling and tackling the social factors often associated with unhealthy lifestyles would support health promotion at community level. Ongoing research will allow comparison and development of innovative approaches to clinical assessment and health promotion.

\section{THE FUTURE: PROFESSIONAL COOPERATION}

The usefulness of clinical assessment in general practice depends on its integration into the everyday activities of the practice. A sophisticated level of cooperation between the GP and other health professionals either in the practice or accessible in the community, will be essential in order to implement the necessary follow-through to disease prevention and health promotion. These health professionals include practice nurses, dietitians, physiotherapists, psychologists, food and cookery advisors, public health nurses and public health physicians who can assist the public in making appropriate lifestyle changes. To date, our 
primary-care services have been underdeveloped in utilizing the skills of these health professionals. With the increasing emphasis on disease prevention and health promotion in the health services, as signalled by the Department of Health $(1994,1995 a)$ in the health strategy and the strategy for health promotion documents, the time has come for the integration of a comprehensive range of health care skills into primary care. As health professionals, we must find the best methods to communicate our message, be open to new ideas and assist our clients in realizing their own need for change. Clinical assessment provides a good basis from which to commence disease prevention and health promotion for the individual. It also provides a starting point for professional cooperation in primary care.

The author wishes to acknowledge the inspiration of the late Professor Noel Hickey in setting up CRISP; also to acknowledge the support of her colleagues in the Department of Preventive Medicine/Cardiology and the Department of Nutrition and Dietetics, St Vincent's Hospital, Dublin. Special thanks to Ms Sheena Rafferty who was the CRISP dietitian from March 1993 to February 1995 and to Ms Colette O'Brien for patiently typing this paper.

\section{REFERENCES}

Cupples, M. E. \& McKnight, A. (1994). Randomised controlled trial of health promotion in general practice for patients at high cardiovascular risk. British Medical Journal 309, 993-996.

Department of Health (1994). Shaping a Healthier Future. A Strategy for Effective Healthcare in the 1990s. Dublin: Stationery Office.

Department of Health (1995a). A Health Promotion Strategy. Dublin: Department of Health

Department of Health (1995b). Recommendation for a Food and Nutrition Policy for Ireland. Dublin: Department of Health.

Family Heart Study Group (1994). Randomized controlled trial evaluating cardiovascular screening and intervention in general practice: principal results of British family heart study. British Medical Journal 308, $313-320$.

Field, K., Thorogood, M., Silagy, C., Normand, C., O'Neill, C. \& Muir, J. (1995). Strategies for reducing coronary risk factors in primary care: which is most cost effective? British Medical Journal 310, 1109-1112.

Gill, T. (1995). Optimism on OXCHECK. Correspondence. Practice Nurse 9, 632.

Gleeson, T. \& Flynn. M. (1990). A dietitian - who needs one? Irish Doctor 3, 169-172.

Grover, S. A., Lowensteyn, I., Esrey, K. L., Steinert, Y., Joseph, L. \& Abrahamowicz, M. (1995). Do doctors accurately assess coronary risk in their patients? British Medical Journal 310, 975-978.

Imperial Cancer Research Fund OXCHECK Study Group (1995). Effectiveness of health checks conducted by nurses in primary care: final results of the OXCHECK Study. British Medical Journal 310, 1099-1104.

Irish Heart Foundation (1994). Happy Heart National Survey. Dublin: Irish Heart Foundation.

Kilkenny Health Project (1992). A Pilot Programme for Coronary Heart Disease Prevention, 1985-1992. Kilkenny, Republic of Ireland: Health Promotion Unit, South Eastern Health Board.

Lindholm, L. H., Ekbom, T., Dash, C., Eriksson, M., Tibblin, G. \& Schersten, B., on behalf of the CELL Study Group (1995). The impact of health care advice given in primary care on cardiovascular risk. British Medical Journal 310, 1105-1109.

Marteau, T. M., Kinmonth, A.-L., Pyke, S., Thompson, S. G. (Family Heart Study Group) (1995). Readiness for lifestyle advice: self-assessments of coronary risk prior to screening in the British family heart study. British Journal of General Practice 45, 5-8.

Pyorala, K., De Backer, G., Graham, I., Poole-Wilson, P. \& Wood, D., on behalf of the Task Force of the European Society of Cardiology, European Atherosclerosis Society and European Society of Hypertension (1994). Prevention of coronary heart disease in clinical practice. European Heart Journal 15, 1300-1331.

Silagy, C., Muir, J., Coulter, A., Thorogood, M. \& Roe, L. (1993). Cardiovascular risk and attitudes to lifestyle: what do patients think? British Medical Journal 306, 1657-1660.

Toon, P. (1995). Editorial: Health checks in general practice - time to review their role. British Medical Journal 310, 1083-1084.

Printed in Great Britain 